Hamam Abd* and Andreas König

\title{
Adaptive Spiking Sensor System Based on CMOS Memristors Emulating Long and Short-Term Plasticity of Biological Synapses for Industry 4.0 Applications
}

\author{
Adaptives Spiking-Sensorsystem auf Basis von CMOS-Memristoren, das die lang- und kurzfristige \\ Plastizität biologischer Synapsen für Industrie 4.0-Anwendungen emuliert
}

\section{DOI 10.1515/teme-2021-0057}

\begin{abstract}
A conventional analog to digital converter (ADC) faces many issues with leading-edge technologies due to noise, manufacturing deviations, signal swings, etc. Thus, we pursue to design an adaptive spiking neural ADC (SN-ADC) with promising features, e.g., robust to noise, low-power, technology scaling issues, and low-voltage operation. Therefore, our approach promises to be technology agnostic, i.e., effectively translatable to aggressive new technologies. It supports machine learning and self-x (selfcalibration, self-healing) that needs for industry 4.0 and the internet of things (IoTs). In this work, we design an adaptive spike-to-rank coding (ASRC), which is the main part of the spiking neural ADC. The ASRC is based on CMOS memristors emulating short-term plasticity (STP) and long-term plasticity (LTP) biological synapses. The proposed ASRC compensates deviations by adapting the weights of the synapses. Also, ASRC is designed using XFAB $0.35 \mu \mathrm{m}$ CMOS technology and Cadence design tools. In addition, ASRC is simulated to test its performance in the temperature range $\left(-40^{\circ} \mathrm{C}\right.$ to $\left.85^{\circ} \mathrm{C}\right)$.
\end{abstract}

Keywords: Adaptive spiking sensor electronics system, Spiking neural networks, Adaptive synapse, Industry 4.0, CMOS Memristor, Self-x, Memristor emulator, Internet of Things (IoT), Machine learning, Memristor neuromorphic architectures.

Zusammenfassung: Ein konventioneller Analog-DigitalWandler (ADC) hat mit Spitzentechnologien viele Probleme aufgrund von Rauschen, Fertigungsabweichungen,

\footnotetext{
*Corresponding author: Hamam Abd, Kognitive Integrierte Sensorsysteme (KISE), TU Kaiserslautern, Deutschland; and College of Electronics Engineering, Ninevah University, Iraq, E-Mail: abd@eit.uni-kl.de
}

Andreas König, Kognitive Integrierte Sensorsysteme (KISE), TU Kaiserslautern, Deutschland
Signalschwankungen usw. Daher verfolgen wir das Ziel, einen adaptiven Spiking Neural ADC (SN-ADC) mit vielversprechenden Eigenschaften zu entwerfen, z. B. robust gegenüber Rauschen, geringem Stromverbrauch, Skalierungsproblemen der Technologie und Betrieb mit niedriger Spannung. Daher verspricht unser Ansatz, technologieunabhängig zu sein, d.h. effektiv auf aggressive neue Technologien übertragbar. Er unterstützt maschinelles Lernen und Self-x (Selbstkalibrierung, Selbstheilung), die für Industrie 4.0 und das Internet der Dinge (IoT) benötigt werden. In dieser Arbeit entwerfen wir eine adaptive Spike-to-Rank-Codierung (ASRC), die der Hauptbestandteil des spikenden neuronalen ADC ist. Die ASRC basiert auf CMOS-Memristoren, die biologische Synapsen mit Kurzzeitplastizität (STP) und Langzeitplastizität (LTP) emulieren. Der vorgeschlagene ASRC kompensiert Abweichungen, indem er die Gewichte der Synapsen anpasst. Die ASRC wird in XFAB 0,35 $\mu \mathrm{m}$ CMOS-Technologie und mit Cadence-Design-Tools entworfen. Darüber hinaus wird ASRC simuliert, um seine Leistungsfähigkeit im Temperaturbereich $\left(-40^{\circ} \mathrm{C}\right.$ bis $\left.85^{\circ} \mathrm{C}\right)$ zu testen.

Schlüsselwörter: Adaptive Spiking-Sensorelektronik, Spiking neuronale Netze, Adaptive Synapse, Industrie 4.0, CMOS-Memristor, Self-x, Memristor-Emulator, Internet of Things (IoT), Maschinelles Lernen, Memristor neuromorphe Architekturen.

\section{Introduction}

In the last few years, the advances in machine learning and self-x in industry 4.0, as well as IoT, have led to an increment in the number of edge devices [3, 18, 38]. Modern devices are still using amplitude coded signals information processing which is vulnerable to noise with down-scaling technologies. Time or spike domain information process- 
ing is more robust to noise [10]. The memristor can push new computing systems beyond Moore's law [26, 42]. Biologically inspired systems can significantly benefit from memristor [23, 40, 42]. This concept assists to overcome the hurdles faced in recent cognitive integrated sensor systems, biologically inspired systems, and low-power intelligent systems [42]. The memristor spiking neural network (SNN) architecture offers noise-robustness, technology agnostic robustness, small on-chip footprint, scalability, low power, machine learning, and self-x properties, needed for industry 4.0, as well as IoT $[17,19,21,27,36]$. Adaptive synapses are the element key of the SNN.

Many researchers have focused on design emulating biological synapses to design a neuromorphic system that can work similarly to biological neural network sensors $[6,7,9,14,28,37]$. Recently, many researchers focused on obtaining the synaptic functions with memristors $[16$, 29]. Two types of synaptic plasticity were observed in biological synapses, short-term plasticity (STP) and longterm plasticity (LTP) [12, 25]. In [15, 22, 24, 32, 34, 39], the authors constructed memristors that emulate STP and LTP characteristics of the synapses.

Implementing a spiking neural network (SNN) based on memristor as a synapse inspired by biological neural networks sensors has several challenges such as [19, 41] resistance level stability, the fabrication complexity of memristor systems, the compatibility with CMOS technology, the finite number of resistance levels, reliability and lifetime of memristor devices. Many researchers have concentrated on memristor emulators to implement neuromorphic architectures on contemporary chips $[1,5,8,13,35]$. State-ofthe-art designs can emulate the biological synapses STP of $[5,8,13,20,30,35]$. They can emulate the biological synapses LTP using a big external capacitor, DAC, and external memory. They separated memory and information processing, which led to the limitation of the von Neumann computing architecture. Most of them have complex circuits and use a lot of passive elements and active components.

\section{Jeffress Model Adoption}

A sensory systems approach based on human hearing acoustic localization can be used for time-coded ADCs, theorized by Jeffress [4]. Delay lines in acoustic localization are a lucid example of adaptive SNN, as shown in Figure 1. The location is determined by the brain using the time difference of the sound reaching the left and right ears $\left(t_{1}-t_{2}\right)$ as shown in Figure $1(A)$. The design of the ADC inspired by neural processing in the human auditory system requires two parts. Those are sensor-tospike converter (SSC) and spike-to-digital converter (SDC) [11], as shown in the Figure 1 (B).

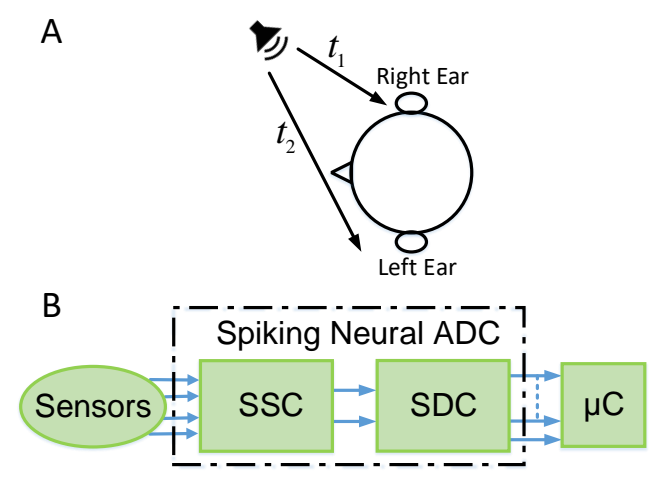

Fig. 1: (A) Acoustic localization model. (B) Neuromorphic signal conditioning system.

The SSC converts the sensor signal to the spikes with the time difference. The SDC generates digital code depending on the time difference between the spikes. The former design of ADC [11] did not make use of adaptivity as required, e.g., to deal with challenges imposed by leading edge technologies. The primary objective of this paper is to design the main part of the SDC. It is an adaptive spike to rank coding (ASRC) based on CMOS memristors emulating long and short-term plasticity of biological synapses. This adaptive design servces as a plateform for machine learning to introduce self-x properties for industry 4.0 applications and IoTs.

\section{Proposed Adaptive SN-ADC}

In this paper, we propose an adaptive synapse based on the CMOS memristor shown in Figure 2. It emulates the STP and LTP of biological synapses. Transistors from M1 to M4 represent the STP of the synapse, and M5 to M12 transistors represent the LTP of the synapse. M4 is functioning as a capacitor by tying the source, drain, and bulk to the ground. The voltage across M4 is controlled by the feedback circuit M2 and M3 transistors. The 8-bit up-down counter is designed using eight JK flip-flops, and this counter changes the LTP of the synapse.

The essential part of the ASRC is the adaptive coincidence detection (ACD) designed by using two adaptive synapses and one neuron, as shown in Figure 3. 


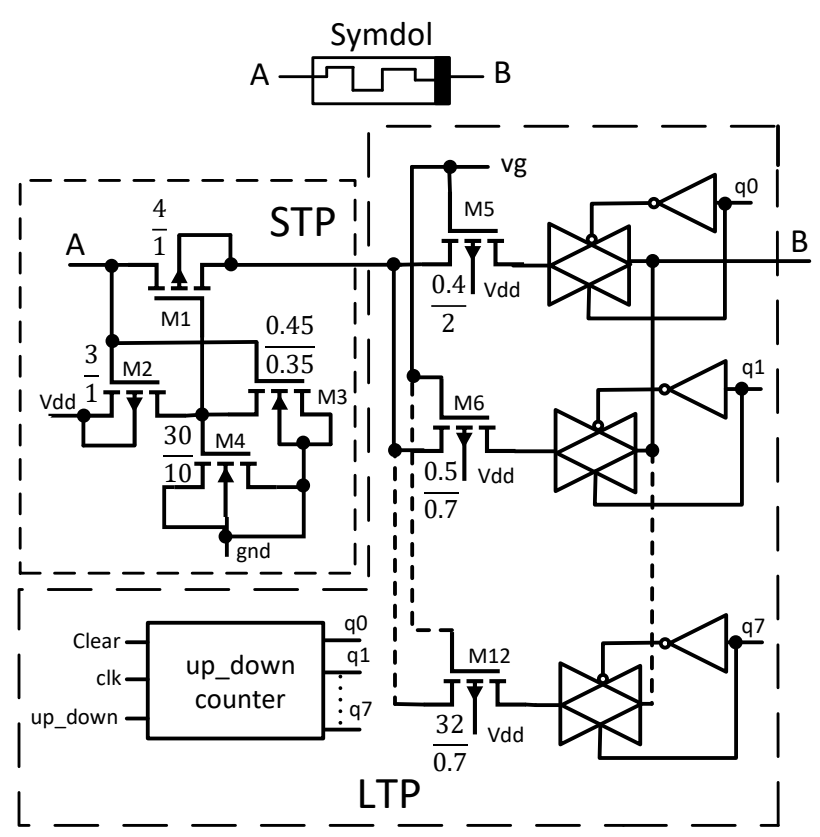

Fig. 2: Proposed adaptive synapse (dimensions of the transistors are in $\mu \mathrm{m})$.

Leaky-integrate-and-fire (LIF) neuron is proposed using memristor in $[31,33]$. In our prior work, we proposed LIF neurons using emulated memristor. For this work, we adjust the previous neuron to serve for the design of the ACD shown in Figure 4. In this figure, $\mathrm{C}$ is the membrane capacitor. The neuron fires when the membrane capacitor voltage is above the threshold voltage of the inverter. The delay line is used to limit the width of the spike and discharge the membrane capacitor. The delay line is implemented using inverters chain due to its simplicity, whilst other possibilities are also feasible for implementation of the functionality [2]. The proposed ASRC demonstrative implementation using eight ACDs is shown in Figure 5.

\section{Results and Discussion}

The proposed ACD emulates the three states that occur in biological neural networks as shown in Figure 6. In the first state, the output spike is generated when both input spikes arrive at the same moment. The second state causes an output spike delayed compared to the previous state if one input spike is shortly delayed than the other. The third state shows no spike if the two inputs spikes reach with a longer delay to each other.

As depicted in Figure 7, the difference between in 1 and in2 has been changed from 0 ns to $60 \mathrm{~ns}$. The width of the input pulse is set to $10 \mathrm{~ns}$ as an optimum value to reach the

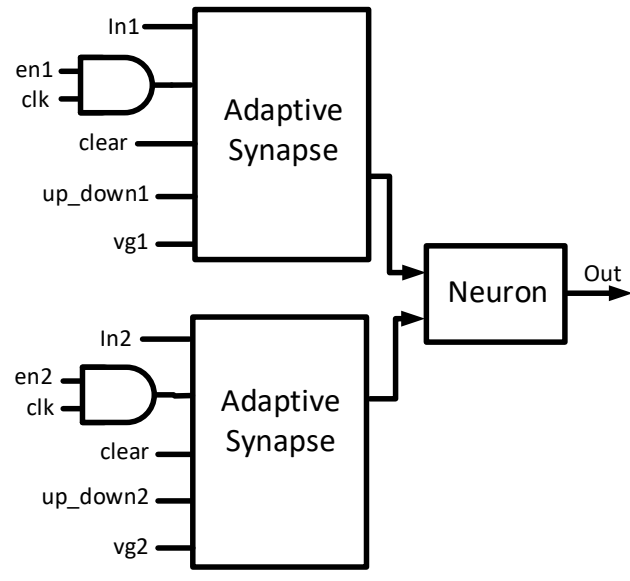

Fig. 3: Proposed adaptive coincidence detection.

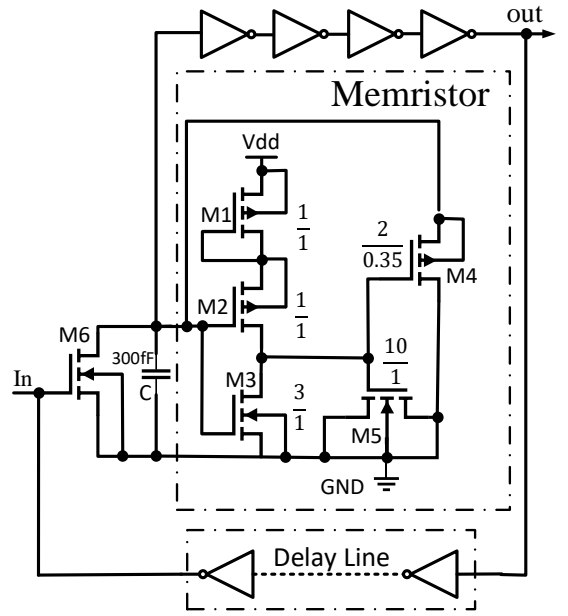

Fig. 4: Proposed neuron based on CMOS memristor (dimensions of the transistors are in $\mu \mathrm{m}$ ).

highest possible speed with less power consumption and minimal capacitor size. The implemented ASRC achieves up to five different output spikes orders, which represents 2.5-bit resolution as shown in Table 1. The resolution can be increased by adding four ACDs for each bit. The number of bits $(\mathrm{NoB})$ can be calculated as:

$$
N o B=\frac{\text { Number of } A C D}{4}+0.5
$$

while the maximum bit-width (MNoB) of the ASRC can be calculated as:

$$
M N o B=\frac{\text { Time Span of } S S C}{2 \times \text { Offset of } S D C}
$$

Where the offset of the SDC is 7.5 ns. Hence a wider and linear span provides more resolution, which is currently under progress. The ASRC is tested under tempera- 
tures $\left(-40{ }^{\circ} \mathrm{C}, 85^{\circ} \mathrm{C}\right)$ to confirm its robustness as presented in Figure 8 and Figure 9.

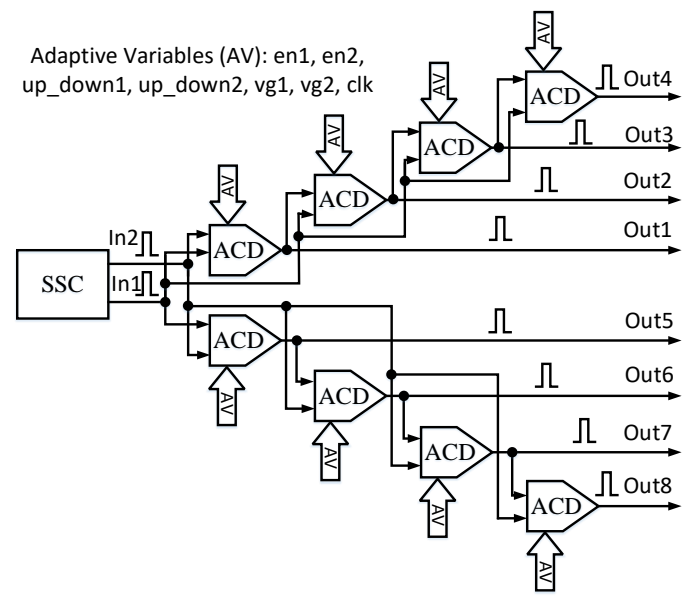

Fig. 5: Proposed adaptive spike-to-rank coding.

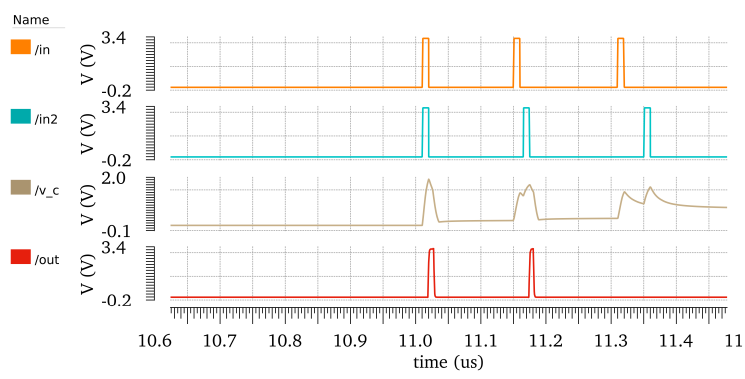

Fig. 6: Simulation of ACD.

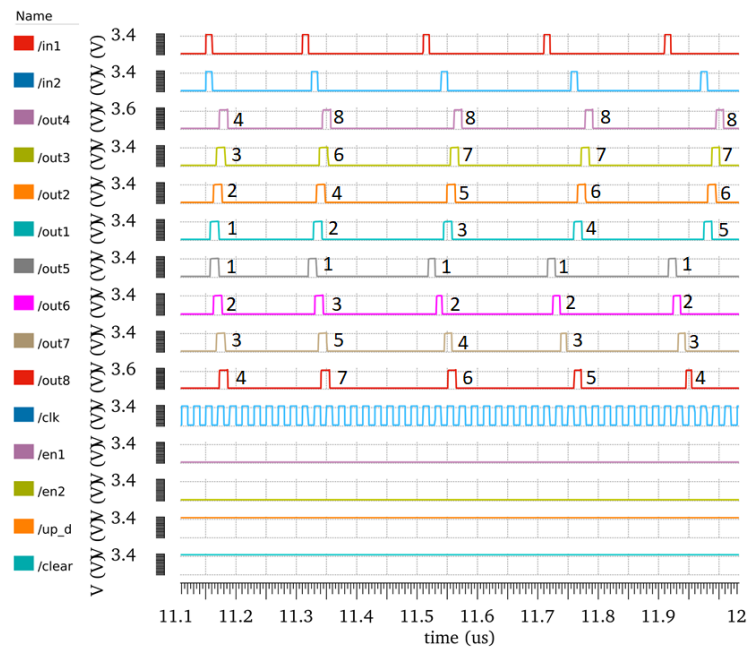

Fig. 7: Rank coding simulation of ASRC at $27^{\circ} \mathrm{C}$.
The output spikes orders are changing with the temperature variations as shown in Table 1 . The weights of the synapses are adapted using up_down1, up_down2, en1, en2, vg1, vg2 variables to compensate for the deviations and reset the output spikes orders to their original states as shown in Figure 10. Similarly, the design adapts for the process and voltage variation to correct output order.

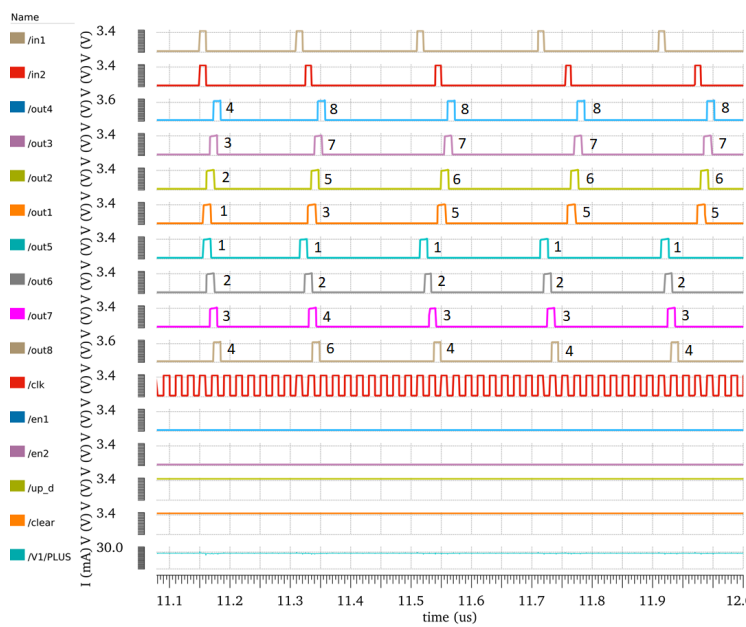

Fig. 8: Rank coding simulation of ASRC at $-40^{\circ} \mathrm{C}$.

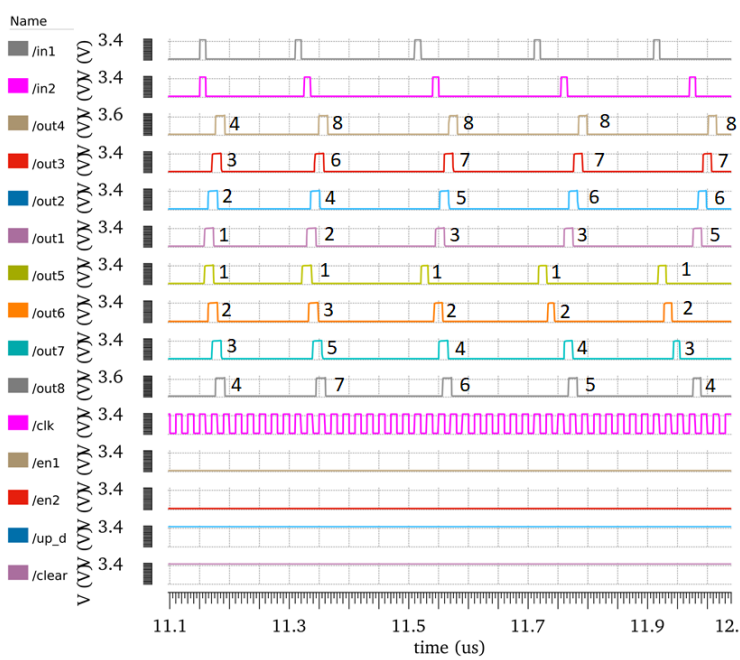

Fig. 9: Rank coding simulation of ASRC at $85^{\circ} \mathrm{C}$.

The ACD consumes energy of 0.99 pJ per 1 us when there is no spike, while it consumes 18.744 pJ per spike. On the other hand, the ASRC consumes an energy of 133.2 pJ per conversion. 
Table 1: Output spike orders of the ASRC at $27{ }^{\circ} \mathrm{C},-40{ }^{\circ} \mathrm{C}$ and $85^{\circ} \mathrm{C}$.

\begin{tabular}{|c|c|c|c|c|c|c|c|c|c|c|c|c|c|c|c|}
\hline \multirow{2}{*}{$\begin{array}{l}\text { Output spike orders } \\
\text { Temperature }{ }^{\circ} \mathrm{C}\end{array}$} & \multicolumn{3}{|c|}{$1^{\mathrm{st}}$} & \multicolumn{3}{|c|}{$2^{\text {nd }}$} & \multicolumn{3}{|c|}{$3^{\text {rd }}$} & \multicolumn{3}{|c|}{$4^{\text {th }}$} & \multicolumn{3}{|c|}{$5^{\text {th }}$} \\
\hline & 27 & -40 & 85 & 27 & -40 & 85 & 27 & -40 & 85 & 27 & -40 & 85 & 27 & -40 & 85 \\
\hline Out1 & 1 & 1 & 1 & 2 & 3 & 2 & 3 & 5 & 3 & 4 & 5 & 3 & 5 & 5 & $\overline{5}$ \\
\hline Out2 & 2 & 2 & 2 & 4 & 5 & 4 & 5 & 6 & 5 & 6 & 6 & 6 & 6 & 6 & 6 \\
\hline Out3 & 3 & 3 & 3 & 6 & 7 & 6 & 7 & 7 & 7 & 7 & 7 & 7 & 7 & 7 & 7 \\
\hline Out4 & 4 & 4 & 4 & 8 & 8 & 8 & 8 & 8 & 8 & 8 & 8 & 8 & 8 & 8 & 8 \\
\hline Out5 & 1 & 1 & 1 & 1 & 1 & 1 & 1 & 1 & 1 & 1 & 1 & 1 & 1 & 1 & 1 \\
\hline Out6 & 2 & 2 & 2 & 3 & 2 & 3 & 2 & 2 & 2 & 2 & 2 & 2 & 2 & 2 & 2 \\
\hline Out7 & 3 & 3 & 3 & 5 & 4 & 5 & 4 & 3 & 4 & 3 & 3 & 4 & 3 & 3 & 3 \\
\hline Out8 & 4 & 4 & 4 & 7 & 6 & 7 & 6 & 4 & 6 & 5 & 4 & 5 & 4 & 4 & 4 \\
\hline
\end{tabular}

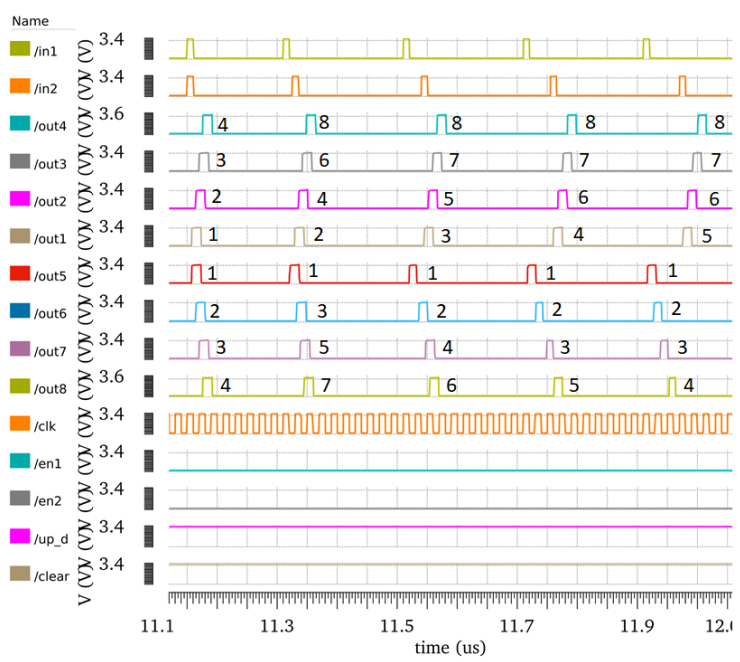

Fig. 10: The output spike orders recovered to their original statuses at $85{ }^{\circ} \mathrm{C}$.

\section{Conclusions and Future Work}

In this work, we presented the design of the ASRC, which is the main part of the adaptive spiking neural ADC. An adaptive synapse based on a CMOS memristor is being proposed to build the ASRC. It emulates the STP and LTP of a biological synapse. In contrast to the previous design, the current ASRC design compensates for the deviations by adapting the weight of the synapse. Additional four ACDs can increase the resolution by one bit, and the maximum bit-width of the ASRC can be calculated using Equation 2. It will be later complemented by the SSC time to get a comparable figure to traditional amplitudebased ADC. The conversion time of the ASRC is $200 \mathrm{~ns}$. The proposed ASRC is implemented using XFAB $0.35 \mu \mathrm{m}$ CMOS technology and tested in the temperature range $\left(-40^{\circ} \mathrm{C}\right.$ to $\left.85^{\circ} \mathrm{C}\right)$ using Cadence design tools. The output spikes orders vary with the process, voltage and temperature changing and are reset to their original states by adapting the weights of the synapses. We are planning to increase the resolution and design the remaining parts of the spiking neural ADC in future work. Moreover, our future research intention is to consider closed synaptic adaptation loops without bulky conventional digital components/units.

\section{References}

[1] H. Abd und A. König. A compact four transistor cmos-design of a floating memristor for adaptive spiking neural networks and corresponding self- $x$ sensor electronics to industry 4.0. tm-Technisches Messen, 87(s1):s91-s96, 2020.

[2] B. I. Abdulrazzaq, I. A. Halin, S. Kawahito, R. M. Sidek, S. Shafie und N. A. M. Yunus. A review on high-resolution cmos delay lines: towards sub-picosecond jitter performance. SpringerPlus, 5(1):1-32, 2016.

[3] S. Alraho, Q. Zaman und A. König. Reconfigurable wide input range, fully-differential indirect current-feedback instrumentation amplifier with digital offset calibration for self-x measurement systems. tm-Technisches Messen, 2020.

[4] G. Ashida und C. E. Carr. Sound localization: Jeffress and beyond. Current opinion in neurobiology, 2011.

[5] Y. Babacan, A. Yesil und F. Kacar. Memristor emulator with tunable characteristic and its experimental results. AEU-IJEC, 81:99-104, 2017.

[6] R. Berdan, E. Vasilaki, A. Khiat, G. Indiveri, A. Serb und T. Prodromakis. Emulating short-term synaptic dynamics with memristive devices. Scientific reports, 6(1):1-9, 2016.

[7] G. W. Burr, R. M. Shelby, A. Sebastian, S. Kim, S. Kim, S. Sidler, K. Virwani, M. Ishii, P. Narayanan, A. Fumarola et al. Neuromorphic computing using non-volatile memory. Advances in Physics: X, 2(1):89-124, 2017.

[8] H. Cao und F. Wang. Spreading operation frequency ranges of memristor emulators via a new sine-based method. IEEE Transactions on VLSI Systems, 2021.

[9] E. Covi, Y.-H. Lin, W. Wang, T. Stecconi, V. Milo, A. Bricalli, E. Ambrosi, G. Pedretti, T.-Y. Tseng und D. lelmini. A volatile rram synapse for neuromorphic computing. In 2019 26th IEEE ICECS.

[10] W. Guo, M. E. Fouda, A. M. Eltawil und K. N. Salama. Neural coding in spiking neural networks: A comparative study 
for robust neuromorphic systems. Frontiers in Neuroscience, 15:212, 2021.

[11] A. Kammara, A. König et al. Ssdc $\alpha$-inherently robust integrated biomimetic sensor-to-spike-to-digital converter based on peripheral neural ensembles. tm-Technisches Messen, 83(9):531-542, 2016.

[12] E. R. Kandel. From nerve cells to cognition: The internal cellular representation required for perception and action. Principles of neural science, 4:381-403, 2000.

[13] G. Kanyal, P. Kumar, S. K. Paul und A. Kumar. Ota based high frequency tunable resistorless grounded and floating memristor emulators. AEU-IJEC, 2018.

[14] M.-K. Kim und J.-S. Lee. Short-term plasticity and long-term potentiation in artificial biosynapses with diffusive dynamics. ACS nano, 12(2):1680-1687, 2018.

[15] S. Kim, C. Du, P. Sheridan, W. Ma, S. Choi und W. D. Lu. Experimental demonstration of a second-order memristor and its ability to biorealistically implement synaptic plasticity. Nano letters, 15(3):2203-2211, 2015.

[16] S. G. Kim, J. S. Han, H. Kim, S. Y. Kim und H. W. Jang. Recent advances in memristive materials for artificial synapses. Advanced Materials Technologies, 2018.

[17] T. Kim, S. Hu, J. Kim, J. Y. Kwak, J. Park, S. Lee, I. Kim, J.-K. Park und Y. Jeong. Spiking neural network (snn) with memristor synapses having non-linear weight update. Frontiers in computational neuroscience, 15:22, 2021.

[18] A. Koenig. Integrated sensor electronics with self-x capabilities for advanced sensory systems as a baseline for industry 4.0. In Sensors and Measuring Systems; 19th ITG/GMASymposium, S. 1-4. VDE, 2018.

[19] O. Krestinskaya, A. P. James und L. O. Chua. Neuromemristive circuits for edge computing: A review. IEEE transactions on neural networks and learning systems, 31(1):4-23, 2019.

[20] P. Kumar, R. K. Ranjan et al. Resistorless memristor emulator using cfta and its experimental verification. IEEE Access, 2021.

[21] J. Li, Z. Dong, L. Luo, S. Duan und L. Wang. A novel versatile window function for memristor model with application in spiking neural network. Neurocomputing, 405:239-246, 2020.

[22] S. Li, F. Zeng, C. Chen, H. Liu, G. Tang, S. Gao, C. Song, Y. Lin, F. Pan und D. Guo. Synaptic plasticity and learning behaviours mimicked through ag interface movement in an $\mathrm{ag} /$ conducting polymer/ta memristive system. Journal of Materials Chemistry C, 1(34):5292-5298, 2013.

[23] Z. Lv, Y. Zhou, S.-T. Han und V. Roy. From biomaterialbased data storage to bio-inspired artificial synapse. Materials today, 21(5):537-552, 2018.

[24] Z. I. Mannan, H. Kim und L. Chua. Implementation of neuromemristive synapse for long-and short-term bio-synaptic plasticity. Sensors, 21(2):644, 2021.

[25] S. J. Martin, P. D. Grimwood und R. G. Morris. Synaptic plasticity and memory: an evaluation of the hypothesis. Annual review of neuroscience, 23(1):649-711, 2000.

[26] A. Mehonic, A. Sebastian, B. Rajendran, O. Simeone, E. Vasilaki und A. J. Kenyon. Memristors-from in-memory computing, deep learning acceleration, and spiking neural networks to the future of neuromorphic and bio-inspired computing. Advanced Intelligent Systems, 2020.

[27] R. Midya, Z. Wang, S. Asapu, S. Joshi, Y. Li, Y. Zhuo, W. Song, H. Jiang, N. Upadhay, M. Rao et al. Artificial neu- ral network (ann) to spiking neural network (snn) converters based on diffusive memristors. Advanced Electronic Materials, 5(9):1900060, 2019.

[28] T. Ohno, T. Hasegawa, T. Tsuruoka, K. Terabe, J. K. Gimzewski und M. Aono. Short-term plasticity and longterm potentiation mimicked in single inorganic synapses. Nature materials, 10(8):591-595, 2011.

[29] Y. Park, M.-K. Kim und J.-S. Lee. Emerging memory devices for artificial synapses. Journal of Materials Chemistry, 2020.

[30] C. Sánchez-López, J. Mendoza-Lopez, M. Carrasco-Aguilar und C. Muñiz-Montero. A floating analog memristor emulator circuit. IEEE Transactions on C\&S II, 2014.

[31] P. Stoliar, J. Tranchant, B. Corraze, E. Janod, M.-P. Besland, F. Tesler, M. Rozenberg und L. Cario. A leakyintegrate-and-fire neuron analog realized with a mott insulator. Advanced Functional Materials, 2017.

[32] Z. Wang, S. Joshi, S. E. Savel'ev, H. Jiang, R. Midya, P. Lin, M. Hu, N. Ge, J. P. Strachan, Z. Li et al. Memristors with diffusive dynamics as synaptic emulators for neuromorphic computing. Nature materials, 2017.

[33] Z. Wang, S. Joshi, S. Savel'ev, W. Song, R. Midya, Y. Li, M. Rao, P. Yan, S. Asapu, Y. Zhuo et al. Fully memristive neural networks for pattern classification with unsupervised learning. Nature Electronics, 1(2):137-145, 2018.

[34] L. Wu, H. Liu, J. Lin und S. Wang. Volatile and nonvolatile memory operations implemented in a $\mathrm{pt} / \mathrm{hfo}_{2} / \mathrm{ti}$ memristor. IEEE Transactions on Electron Devices, 2021.

[35] N. Yadav, S. K. Rai und R. Pandey. Novel memristor emulators using fully balanced vdba and grounded capacitor. Iranian Journal of Science and Technology, Transactions of Electrical Engineering, 45(1):229-245, 2021.

[36] J.-Q. Yang, R. Wang, Z.-P. Wang, Q.-Y. Ma, J.-Y. Mao, Y. Ren, X. Yang, Y. Zhou und S.-T. Han. Leaky integrateand-fire neurons based on perovskite memristor for spiking neural networks. Nano Energy, 74:104828, 2020.

[37] Y. Yang, M. Yin, Z. Yu, Z. Wang, T. Zhang, Y. Cai, W. D. Lu und R. Huang. Multifunctional nanoionic devices enabling simultaneous heterosynaptic plasticity and efficient in-memory boolean logic. Advanced Electronic Materials, 2017.

[38] Q. Zaman, S. Alraho und A. König. Low-cost indirect measurement methods for self- $x$ integrated sensory electronics for industry 4.0. tm-Technisches Messen, 2020.

[39] X. Zhang, S. Liu, X. Zhao, F. Wu, Q. Wu, W. Wang, R. Cao, Y. Fang, H. Lv, S. Long et al. Emulating short-term and long-term plasticity of bio-synapse based on cu/a-si/pt memristor. IEEE Electron Device Letters, 2017.

[40] Y. Zhang, Z. Wang, J. Zhu, Y. Yang, M. Rao, W. Song, Y. Zhuo, X. Zhang, M. Cui, L. Shen et al. Brain-inspired computing with memristors: Challenges in devices, circuits, and systems. Applied Physics Reviews, 7(1):011308, 2020.

[41] Y. Zhang, Z. Wang, J. Zhu, Y. Yang, M. Rao, W. Song, Y. Zhuo, X. Zhang, M. Cui, L. Shen et al. Brain-inspired computing with memristors: Challenges in devices, circuits, and systems. Applied Physics Reviews, 7(1):011308, 2020.

[42] M. A. Zidan, J. P. Strachan und W. D. Lu. The future of electronics based on memristive systems. Nature electronics, 1(1):22-29, 2018. 